\title{
An analysis of the train-turnout interaction
}

\author{
Mihaela Cristina Tudorache", Răzvan Andrei Oprea \\ Railway Vehicles Engineering Department, University Politehnica of Bucharest, Romania
}

\begin{abstract}
A distinctive feature of the application proposed in this work is the occurrence of simultaneous contact patches on a single wheel, i.e., the multi-point contact case. A difficulty in the study of this phenomenon is that the contact areas depend on the deformation of the bodies, whereas these deformations are functions of the contact loading and the contact area. This is contrary to what is usually the case in finite element analysis, where the load and displacement are prescribed at different (fixed) parts of the boundary.
\end{abstract}

\section{Introduction}

Crossings points and switches are designed to offer a continuous support for the wheels throughout the crossing, giving a smooth transition. However, high impact loads may arise $[1,2]$, leading to damage, fracture and wear of both the crossing and the wheels and to high maintenance costs. Therefore, investigations of dynamics and damage in a crossing are of great interest. The basis for the prediction of component life is an adequate determination of stresses and strains.

The UIC (International Union of Railways) and railway researchers have been accomplished several computer applications for the study of train-turnout interaction [3]. The common feature is the employment of commercial multi-body or finite element (FE) software.

Multi-body simulation packages like SIMPACK or GENSYS have been used to simulate the vehicle track interaction due to passing over a turnout $[4,5,6,7]$ and in some cases the results were validated through full-scale field tests [8].

The strip method has been used to determine the wheel-rail contact forces in a turnout with the aid of the Hertzian method to model the wheel-rail contact [9]. The rails have also been modeled in the turn-out zone using several Euler-Bernoulli beams [10].

Flexible track model and real-time wheel-rail penetration contact model in NUCARS was employed for the crossing design [11].

Elastic and elastic-plastic finite element models of the contact are investigated with FE software like ABAQUS. The finite element method is not limited by the half-space assumption or applicable to a linear-elastic material model only $[8,12,13]$. The subject regarding the phenomena resulted from the contact between the road wheel and the railway

*Corresponding author: cristi_tudorache2003@yahoo.com 
represents the base for assuring the safety on guidance traveling and the main focus in the development of railway vehicles and it is approached from different directions in the research field.

It is important to be mentioned that up until recently the problem regarding the influence of these contact phenomena resulted from the contact between the wheel and the railway over the safety guidance has been done using simplifying hypothesis, approximation methods which lead to illustrative methods. Thus, in the present paper it will be presented a threedimensional analysis at the level of the point of contact between the wheel and the turn-out switch from the point of view of the tip of the switch, using the Kalker's Contact algorithm. This is an iterative algorithm which offers an output with an extremely good accuracy in the field of railway transportation [14].

By analyzing the literature, it can easily be remarked the necessity for thorough documentation regarding the study of the influence that the wheel-railway contact phenomena has upon the guidance safety in the sense of reducing the wear of the running surface and optimizing the parameters of the wheel-railway contact surfaces.

\section{2. The basic principles of Kalker theory}

The theory of the linear contact developed by Kalker starts from the De Pater [17] idea, based on the fact that for small values of the pseudo-slip effect the slip area is very small, which considers that the entire contact surface is assimilated with an adhesion area. According to this hypothesis, the condition at the extremity is given by the following equations:

$$
\begin{gathered}
0=w_{x, y}=V \cdot\left(v_{x}-\varphi \cdot y, v_{y}+\varphi \cdot x\right)-V \frac{\partial u(x, y)}{\partial x}-\text { within the contact ellipse. } \\
0=p(x, y) \text { - outside the contact ellipse }
\end{gathered}
$$

By integrating the relation (1) results:

$$
g(y)=-V \cdot u(x, y)+V\left(v_{x} x-\varphi \cdot x \cdot y, v_{\mathrm{y}} x-\frac{1}{2} \varphi \cdot x^{2}\right)
$$

where $g(y)$ represents an arbitrary function.

The arbitrary function $g(y)$ is determined from the condition that the traction force must be constant along the border, where the railway and the wheel come into contact. The final result does not respect though the limit value of the friction force at the end limit of the contact surface [15]. This thing is justified by the fact that in the exterior vicinity of the contact limit the bodies materials are free of internal stress.

The traction increases up to the rear edge of the adhesion limit then the normal load is removed and the traction suddenly drops to 0 . Thus, on the edge, the friction index shows a discontinuous, infinite fluctuation. Calculating the $g(y)$ function will lead to a linear expression displaying the interdependence between forces and pseudo-slip:

$$
\begin{aligned}
& F_{x}=-a \cdot b \cdot C_{11} \cdot v_{x}, F_{y}=-a \cdot b \cdot G\left(C_{22} \cdot v_{y}+\sqrt{a \cdot b} \cdot C_{23} \cdot \varphi\right) \\
& M_{\varphi}=-\left(\sqrt[3]{a \cdot b} \cdot G \cdot C_{32}+(a \cdot b)^{2} \cdot G \cdot C_{33} \cdot \varphi\right)
\end{aligned}
$$

where: $a, b$ - semi-axes of the contact ellipse, $v_{\mathrm{x}}, v_{\mathrm{y}}, \varphi$ - longitudinal, transversal and spinning pseudo-slip movements in the contact points, $C_{11}, C_{22}, C_{23,32}, C_{33}$ - Kalker parameters which 
depend on the value of Poisson index and being determined based on the following equations $[15,16]$ :

$$
\begin{aligned}
& C_{11}=-\frac{\partial F_{x}}{\partial v_{x}} /(a \cdot b \cdot G), \mathrm{C}_{22}=-\frac{\partial F_{y}}{\partial v_{y}} /(a \cdot b \cdot G) \\
& C_{23}=-\frac{\partial F_{y}}{\partial \varphi} /(\sqrt[3]{a \cdot b \cdot G})_{v_{x}=v_{y}=\varphi=0}
\end{aligned}
$$

Kalker defines the pseudo-slip parameters as following:

$$
k_{x}=\frac{a \cdot b \cdot G \cdot C_{11} \cdot v_{x}}{\mu \cdot N}, k_{y}=\frac{a \cdot b \cdot G \cdot C_{22} \cdot v_{y}}{\mu \cdot N}, k_{\varphi}=\frac{\sqrt[3]{a \cdot b} \cdot G \cdot C_{23} \cdot \varphi}{\mu \cdot N}
$$

The longitudinal, transversal and spinning pseudo-slip movements are established based on the following equations:

$$
v_{x}=\frac{w_{x}}{V}, \quad v_{\mathrm{y}}=\frac{w_{y}}{V}, \varphi=\frac{\mathrm{r} \cdot \omega_{\mathrm{s}}}{\mathrm{V}}
$$

where: $\omega_{\mathrm{s}}$ is the spinning angle speed. The pseudo-slip speeds $w_{x, y}$ are established based on the (1) relation.

\section{Numerical application}

Within this section, we will be analyzing the behavior of an axle equipped with wheels having the S1002 profile (the nominal rolling radius is of $460 \mathrm{~mm}$ ) over a simple railroad siding made of a rail track profile type UIC 60, 1:20 and 1:40. The speed with which the axle will pass over the railroad siding is of $35 \mathrm{~m} / \mathrm{s}$. The contact point is analyzed in a three-dimensional reference frame. The normal load applied on the wheel is considered to be of $10 \mathrm{kN}$. For evaluating the behavior between wheel and the rail tongue an iterative program has been developed within the Kalker CONTACT frame, applied for the dual-contact analysis. As a result, the calculus stages which mention the input data for the CONTACT program are the following, $[17,18]$ :

1. Determining the coordinates of the first point of contact through the method of the minimum height difference.

2. The calculus of the penetration within the hypothesis of a single contact point.

3. Verifying the possibility of the development of a new contact area. If this is possible the previous two stages are re-iterated.

4. Determining the penetrations within the contact areas.

5.Re-compute the values for the vertical components (through the equations shown below). If the values obtained here show differences compared to the values obtained at step 4 , differences which are not within the accepted margins, then the calculus at step 4 is reperformed again with vertical components determined at step 5 up until the results obtain are within the imposed limits.

By analyzing the influence that an inclination of the tip of the turn-out switch has over the phenomena from within the contact are we can observe that the grip area is considerably enlarged in the case of an inclination of 1:20 (see Fig. 1a), when comparing that with an inclination of 1:40 (see Fig. 1b). This effect appears only in the contact point from the edge of the wheel, where the guidance is achieved. At the point from the rolling surface or the leaning point the effect is insignificant; here only the sliding surface counts. 

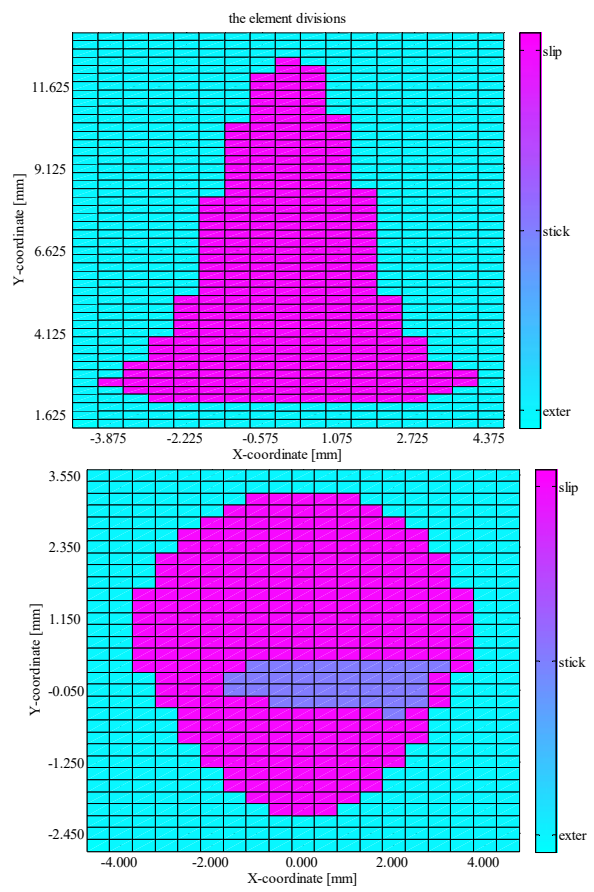
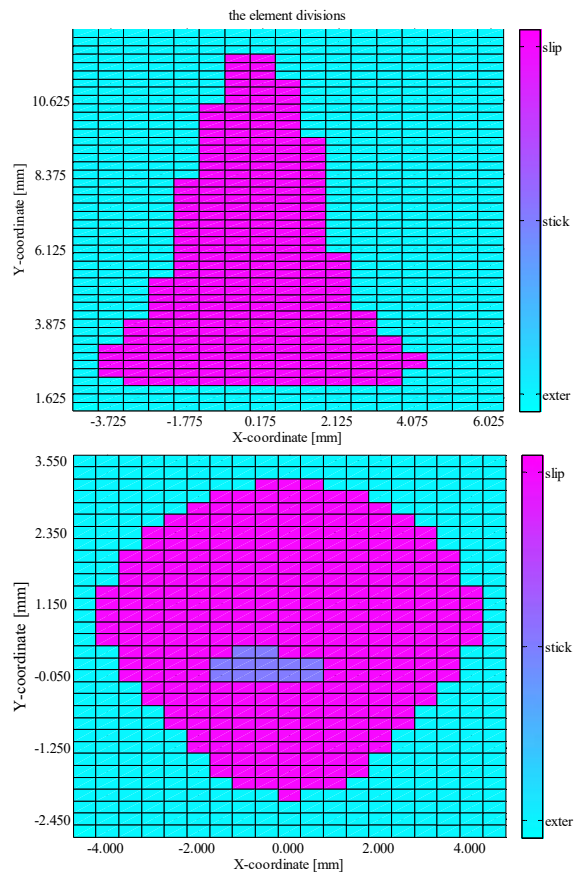

b

Fig. 1. The distribution of the adherence and sliding zones in the two contact points, for different inclinations of the turn-out switch tip: 1 - 1:20, $\mathrm{b}-1: 40$.
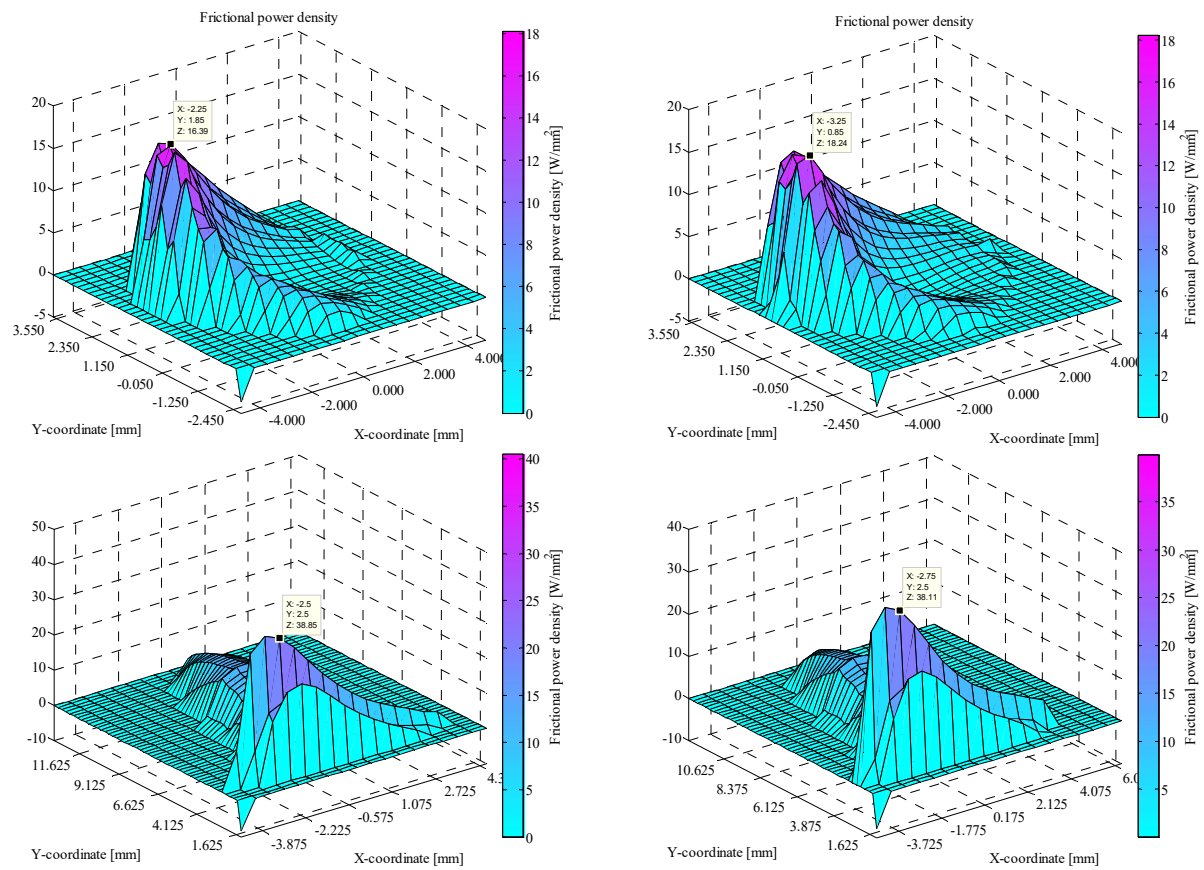

Fig. 2. The density of the power of friction, for different inclinations of the turn-out switch tip: left 1: 20 , right $-1: 40$. 
We can assert that there is an elongation of the shape of the contact surface for the 1:20 inclination, a fact clearly visible in both points and justified by a better guidance. This assertion is reasoned by the fact that for the wear profiles the larger half-axis of the contact surface is orientated perpendicularly on the railway.

According to Fig. 2 the wear in the contact points differs from one inclination to the other. The density of the friction power is increasing in the fulcrum (rolling surface) for the 1:20 inclination and it is decreasing on the wheel's edge, thus assuring a good reliability of the wheel and of the turn-out switch tip.

In the end the effect resulting from the inclination of the turn-out switch tip is reflecting over the guiding transverse forces; for the 1:20 inclination a smaller value is obtained in comparison with a 1:40 inclination $1: 40,(458 \mathrm{~N} / 498 \mathrm{~N})$.

The application is designed for frictional concentrated contact problems. The solution is numerically efficient and it has been previously validated for the general case of the wheelrail contact.

Multi-point contact is a common situation for the studied system. In the approach proposed, the bodies deformations and the contact areas can be determined from the contact loading. Further, the number and position of the contact points can be calculated either online or off-line. The scheme proposed also allows the computation of the loading and stress state starting from the local deformations.

\section{References}

1. E. Kassa, Simulation of Dynamic Interaction between Train and Turnout, Lic. Thesis, Chalmers University of Technology (Department of Applied Mechanics, Gothenburg, Sweden, 2004)

2. M. Wiest, W. Daves, F.D. Fischer, H. Ossberger, Deformation and damage of a crossing nose due to wheel passages, Wear 265, 9-10 (2008)

3. Y.Q. Sun, C. Cole, M. McClanachan, The Calculation of Wheel Impact Force Due to the Interaction between Vehicle and a Turnout, Proceedings of the Institution of Mechanical Engineers, Part F: Journal of Rail and Rapid Transit 1 (2010)

4. G. Schupp, C. Weidemann, and L. Mauer, Modelling the contact between wheel and rail within multibody system simulation, Veh. Syst. Dyn., 415 (2004)

5. G. Schupp, Simulation of railway vehicles: necessities and applications, Mech. Based Des. Struct. Mach. 313 (2003).

6. E. Kassa, C., Andersson, and J.C.O. Nielsen, Simulation of dynamic interaction between train and railway turnout, Veh. Syst. Dyn. 443 (2006)

7. E. Kassa, J.C.O., Nielsen, Dynamic interaction between train and railway turnout: fullscale field test and validation of simulation models, Veh. Syst. Dyn. 465 (2008)

8. E. Kassa, J.C.O. Nielsen, Stochastic analysis of dynamic interaction between train and railway turnout, Veh. Syst. Dyn. 465 (2008)

9. E. Kassa, J.C.O. Nielsen, Dynamic train-turnout interaction in an extended frequency range using a detailed model of track dynamics, J. Sound Vibr. (2009)

10. Z. Ren, S. Sun, W. Zhai, Study on lateral dynamic characteristics of vehicle/turnout system, Veh. Syst. Dyn., 434 (2005)

11. X. Shu, N. Wilson, C. Sasaoka, J. Elkins, Development of a real-time wheel/rail contact model in NUCARS and application to diamond crossing and turnout design simulations, Veh. Syst. Dyn., 44 (Suppl) (2006).

12. C. Andersson, T. Dahlberg, Wheel/rail impacts at a railway turnout crossing. Proc. IMechE, Part F: J. Rail and Rapid Transit, 212(F2) (1998)

13. M. Wiest, W. Daves, J.C.O. Nielsen, H. Ossberger: Assessment of methods for calculating contact pressure in wheel-rail/switch contact, Wear 265 9-10 (2008) 
14. E.A.H. Vollebregt, User guide for CONTACT, Vollebregt \& Kalker's rolling and sliding contact model, VORtech Computing the Scientific Software Engineers (2012)

15. J.J. Kalker, Wheel-rail rolling contact theory, Wear 144 (1991)

16. I. Sebeşan, Railway Vehicles Dynamics (MatrixRom, 2011)

17. C. Tudorache, R. Oprea, A method for studying the interaction of wheel-turnout multipoint contact, Scientific Bulletin - University Politehnica of Bucharest, Series D: Mechanical Engineering 771 (2015)

18. M. C. Tudorache, The influence of wheel-rail phenomena upon guiding safety for railway vehicle, $\mathrm{PhD}$ Thesis (Bucharest 2013) 